\title{
Current exposure of Italian women of reproductive age to PFOS and PFOA: A human biomonitoring study
}

\author{
Elena De Felip ${ }^{a}$, Annalisa Abballe ${ }^{\mathrm{a}}$, Fulvia Lucia Albano ${ }^{\mathrm{b}}$, Tatiana Battista ${ }^{\mathrm{c}}$, Valter Carraro ${ }^{\mathrm{d}}$, \\ Michele Conversano ${ }^{c}$, Silva Franchini ${ }^{\mathrm{d}}$, Laura Giambanco ${ }^{\mathrm{e}}$, Nicola Iacovella ${ }^{\mathrm{a}}$, Anna Maria Ingelido ${ }^{\mathrm{a}, *}$, \\ Antonio Maiorana ${ }^{e}$, Francesco Maneschi ${ }^{\mathrm{f}}$, Valentina Marra ${ }^{\mathrm{a}}$, Antonella Mercurio ${ }^{\mathrm{e}}$, Roberta Nale ${ }^{\mathrm{f}}$, \\ Bianca Nucci $^{g}$, Vincenzo Panella ${ }^{h}$, Flavia Pirola ${ }^{h}$, Maria Grazia Porpora ${ }^{i}$, Enrico Procopio ${ }^{j}$, Nicola Suma ${ }^{j}$, \\ Silvia Valentini ${ }^{\mathrm{a}}$, Luisa Valsenti ${ }^{\mathrm{k}}$, Valerio Vecchiè ${ }^{\mathrm{g}}$
}

a Istituto Superiore di Sanità, Dipartimento Ambiente e connessa Prevenzione Primaria, Roma, Italy

b Ospedale Ostetrico Ginecologico Sant'Anna, Città della Salute e della Scienza, Torino, Italy

${ }^{\mathrm{c}}$ ASL Taranto, Dipartimento Prevenzione, Taranto, Italy

d Azienda Provinciale per i Servizi Sanitari della Provincia Autonoma di Trento, Unità Operativa Igiene e Sanità Pubblica, Trento, Italy

e Azienda di rilievo Nazionale e di Alta Specializzazione, Ospedale Civico di Palermo, U.O.C. di Ginecologia e Ostetricia, Palermo, Italy

${ }_{\mathrm{f}}^{\mathrm{f}}$ Azienda Sanitaria Locale di Latina, P.O. Nord "S. Maria Goretti", U.O.C. Ostetricia e Ginecologia, Latina, Italy

${ }^{g}$ ASL 5 Pinerolo (To), Italy

${ }^{\text {h }}$ ASL Roma D, Roma, Italy

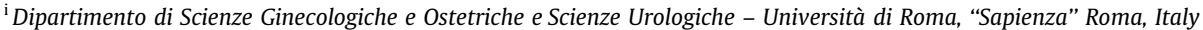

${ }^{\mathrm{j}}$ ASL 5 Susa (To), Italy

${ }^{\mathrm{k}}$ ex ASL 4 Terni (now ASL Umbria 2), U.O.C. Igiene e Sanità Pubblica, Terni, Italy

\section{H I G H L I G H T S}

- Largest human biomonitoring study in women of reproductive age in Italy.

- No differences in PFOS concentrations between urban/industrial and rural areas.

- Levels of PFOA higher in women residing in urban/industrial areas.

- A downward temporal trend in exposure is observed for both compounds.

\section{A R T I C L E I N F O}

\section{Article history:}

Received 17 November 2014

Received in revised form 13 March 2015

Accepted 23 March 2015

Available online 15 May 2015

Handling Editor: Andreas Sjodin

\section{Keywords:}

Biomonitoring

Italy

Women

PFOS

PFOA

\begin{abstract}
A B S T R A C T
Perfluorooctane sulfonate (PFOS) and perfluorooctanoic acid (PFOA) concentrations were determined in serum samples collected in 2011-2012 from 549 nulliparous Italian women of reproductive age who resided in six different Italian Regions. Assessment of exposure to perfluorinated compounds was part of a large human biomonitoring study (Project Life Plus "Womenbiopop") that aimed at examining the exposure of women of reproductive age to priority organic pollutants.

The median concentrations of PFOS and PFOA were 2.43 , and $1.55 \mathrm{ng} \mathrm{g}^{-1}$, respectively.

Significant differences in the concentrations of both compounds were observed among the six Regions. Women from central Italy had the highest levels of both compounds, followed by women from northern Italy, and southern Italy. No differences in the PFOS concentrations were found between women from urban/industrial areas and women from rural areas, whereas the levels of PFOA were significantly higher in women residing in urban/industrial areas than in women residing in rural areas.

Taken together, the observed concentrations confirm that the overall exposure of the Italian population is among the lowest observed in industrialized countries.

A downward temporal trend in exposure was observed for both compounds when comparing the results from the present study with those assessed in a study conducted in 2008.
\end{abstract}

(c) 2015 Elsevier Ltd. All rights reserved.

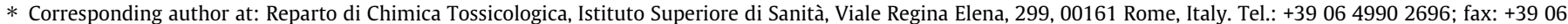
49902836.

E-mail address: annamaria.ingelido@iss.it (A.M. Ingelido). 


\section{Introduction}

The two perfluorinated compounds, perfluorooctane sulfonate (PFOS) and perfluorooctanoic acid (PFOA), represent the most widely studied members of the large family of perfluoroalkylated substances (PFAS). Because of their excellent performance as surfactants and surface protectors, PFOS and PFOA have been extensively used since the 1950 s in a number of applications, including in the surface coatings of cooking pans, in making products water- and oil-repellent, in firefighting foams, and in food packaging. Humans are exposed to PFOS and PFOA principally through their diet (EFSA, 2008a), but ingesting contaminated dust and drinking water may also account for a portion of the exposure (EFSA, 2008a; Wilhelm et al., 2010; Post et al., 2012).

As a consequence of their persistence, bioaccumulation potential, toxicity, and widespread presence in environmental media, biota, and humans, the production and use of these compounds and their precursors have been restricted or banned in several countries. The EU restricted PFOS production and use from 2008 onward (Directive 2006/122/EC of the European Parliament and of the Council). In 2009, PFOS was included in Annex B of the Stockholm Convention on Persistent Organic Pollutants. The toxicity of PFOS and PFOA has been studied quite extensively, and the main toxic effects identified are hepatotoxicity, developmental toxicity, immunotoxicity, and changes in the circulating hormones and lipoproteins (EFSA, 2008b; White et al., 2011; Barouki et al., 2012; DeWitt et al., 2012). Nevertheless, the toxicological profile of these substances is far from being adequately characterized because of the marked differences in toxicokinetics that have been observed among species, which make it difficult to extrapolate the experimental data to humans (Lau, 2012). PFOA is among the substances that are scheduled for IARC evaluation on carcinogenicity this year (IARC, 2008).

PFOS and PFOA were among the persistent organic pollutants (POPs) included in the human biomonitoring project "Womenbiopop - Linking Environment and Health - A Countrybased Study on Women of Reproductive Age" (April 2010-May 2013). This project, funded by the financial instrument "Life Plus" (EC, DG Environment) and co-funded by the Istituto Superiore di Sanità (Italian National Institute of Health) and by the Italian Ministry of Environment, Land and Territory, aimed to characterize the exposure of women of reproductive age to a group of priority POPs of environmental origin, including polychlorodibenzodioxins and polychlorodibenzofurans ("dioxins"), polychlorobiphenyls (PCBs), polybrominated diphenylethers (PBDEs), and some organochlorinated pesticides ( $\beta$-hexachlorocyclohexane, $p, p^{\prime}$-DDE, hexachlorobenzene), and to explore a possible correlation between exposure and women's reproductive health.

PFOS and PFOA were included in the group of POPs that were of interest for the project because of the increasing burden of toxicology and epidemiology studies that have shown a possible impact of these substances on female reproductive health. In particular, a number of animal toxicology studies have shown that exposure to PFOS and PFOA can alter ovarian function (Zhai et al., 2012) and affect the development of mammary gland tissue (White et al., 2011). Epidemiologic research, which exists primarily as cross-sectional studies, on the possible effects on female reproductive health has so far produced inconsistent results. Fei et al. (2009) observed an association between high serum levels of PFOS and PFOA and a longer time to pregnancy, but no such association was found in other studies (Vestergaard et al., 2012; Whitworth et al., 2012). PFOS and PFOA pass through the placenta (Needham et al., 2011) and are excreted in milk, although less so than other POPs (Haug et al., 2011; Mannetje et al., 2012). Effects of in utero exposure to PFAS on the female reproductive functions have also been reported. In a recent study (Kristensen et al., 2013) daughters who were exposed in utero to levels of PFOA that were higher than the reference group showed a later age of menarche.

In the Project "Womenbiopop", 549 nulliparous women were enrolled. These women resided in six different Regions that were representative of northern, central, and southern/insular Italy and in areas that are characterized by different levels of anthropogenic activity. The present paper reports the determinations of PFOS and PFOA that were conducted on women's serum samples.

\section{Methods}

\subsection{Study design and samples}

The regions included in the study were Trentino-Alto Adige and Piemonte (northern Italy); Umbria and Lazio (central Italy); Puglia (southern Italy); and Sicilia (insular Italy).

Areas that had presumed different exposure to POPs were included in all Regions, such as areas with background exposure (rural or mountainous areas with no major industrial settlements) and areas with possible incremental exposure (urban areas and/or areas with industrial settlements). Women were enrolled in the following areas (Fig. 1):

In Trentino Alto-Adige, the town of Trento and two mountainous areas in the Province of Trento (Val di Non and Valsugana, where a steel plant has been operating for the last thirty years).

In Piemonte, the town of Torino and two mountainous areas in the Province of Torino (Val Chisone and Val di Susa, where a steel plant has been operating for decades).

In Umbria, the town of Terni (where a large steel plant has been operating since the end of the 19th century) and country areas in the Province of Terni.

In Lazio, the town of Rome, the town of Latina and country areas in the Province of Latina.

In Puglia, the town of Taranto (where one of the largest steel plants in Europe has been operating since 1960) and country areas in the Province of Taranto.

In Sicilia, the town of Palermo and country areas in the Province of Palermo.

Enrolment was done in the years 2011-2012. Thirty to 50 women were enrolled in each area, for a total of 549 women.

Only nulliparous women (or women who never breastfed) were enrolled, considering that breastfeeding may alter women's body burden.

The women's age was in the range $20-40$ years. All women had resided in the area for at least 10 years.

The Project was approved by local Ethics Committees. Prior to blood drawing, signed informed consent was acquired from each woman, and a questionnaire was administered by medical doctors or trained nurses.

\subsection{Questionnaires}

The questionnaires contained questions on anthropometric and socio-demographic variables (age, weight, height, educational level, employment status) and questions aimed at characterizing sources of exposure to POPs (dietary habits, and lifestyle factors). A section of the questionnaire focused on woman's reproductive health and contained specific questions on a range of diseases/dysfunctions (e.g., infertility, repeated miscarriages, endometriosis, thyroid dysfunctions) for which exposure to POPs has been hypothesized to play a role in their etiology.

The dietary variables investigated were the intake of meat (poultry, beef, pork, lamb, ham and salami), fish and seafood, milk and dairy products (milk, yogurt, cheese), eggs, and vegetables (raw and cooked). Because approximately one half of women 


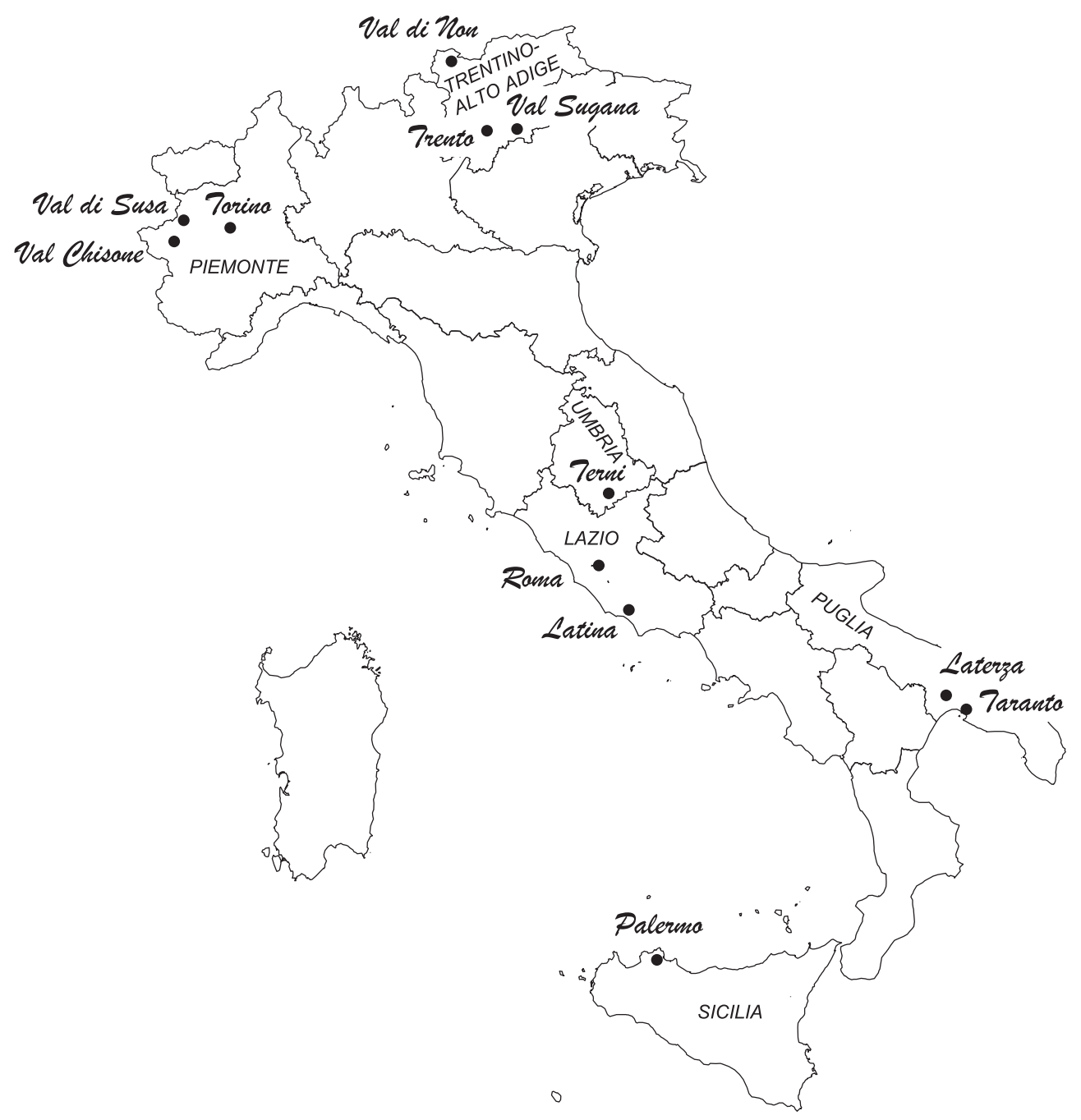

Fig. 1. Sampling areas: Trentino-Alto Adige, Piemonte (northern Italy), Umbria, Lazio (central Italy), Puglia (southern Italy) and Sicilia (insular Italy).

resided in rural or mountainous areas, where the consumption of food of either local or their own production could have accounted for a major fraction of the intake of some food categories, the women were asked to specify the source of purchase (gross distribution or retail vs. local or their own production) for the food categories considered.

\subsection{Analysis}

To analyze all of the POPs considered in the study, approximately $50 \mathrm{~mL}$ of blood was drawn from each woman and centrifuged to obtain serum. An aliquot of approximately $250 \mu \mathrm{L}$ was taken from each serum sample to analyze PFOS and PFOA. The aliquot was fortified with ${ }^{13} \mathrm{C}_{4}$-labelled PFOS and PFOA (Wellington Laboratories Inc., Guelph, Ontario, Canada, purity grade $>99 \%$, $1.150 \mu \mathrm{g} \mathrm{ml}^{-1}$ of each compound), and allowed to rest overnight at $4{ }^{\circ} \mathrm{C}$. Extraction with acetonitrile (Sigma-Aldrich Corp, Saint Louis, MO, USA) was performed in a centrifuge tube. After centrifugation and separation of the two phases, the volume of acetonitrile was reduced and transferred to an autosampler vial to undergo instrumental analysis (Inoue et al., 2004; Ingelido et al., 2010). Instrumental analysis was carried out by HPLC (Waters Alliance 2695, Waters Corporation, Milford, MA, USA) interfaced with a triple quadrupole mass spectrometer (Micromass QuattromicroTM
API, Waters Corporation, Milford, MA, USA) operated in the electrospray negative mode. The working conditions were as follows: Desolvation gas flow, $600 \mathrm{~L} \mathrm{~h}^{-1}$; Cone gas flow, $25 \mathrm{~L} \mathrm{~h}^{-1}$; Source temperature, $120^{\circ} \mathrm{C}$; Desolvation temperature, $450{ }^{\circ} \mathrm{C}$; Capillary voltage, $1 \mathrm{kV}$. The direct injection volume was $30 \mu \mathrm{L}$. Data were acquired using multiple reaction monitoring (MRM). Mass transitions for ${ }^{12} \mathrm{C}$ PFOA were set at $412>369$ and $412>169$, and those for ${ }^{13} \mathrm{C}$ PFOA were set at $417>372$. Mass transitions for ${ }^{12} \mathrm{C}$ PFOS were set at $499>99$ and $499>80$, and those for ${ }^{13} \mathrm{C}$ PFOS were set at $503>99$. The isotope dilution technique was applied throughout the analyses. The recovery ranges were $80-110 \%$ for the ${ }^{13} \mathrm{C}$-labelled internal standards. The limits of quantification for PFOS and PFOA were $0.05 \mathrm{ng} \mathrm{g}^{-1}$ and $0.1 \mathrm{ng} \mathrm{g}^{-1}$, respectively. The concentrations of PFOS were above the limit of quantitation (LOQ) in all samples, and those of PFOA were above LOQ in $98 \%$ of samples.

All analyses were conducted by the laboratory of Toxicological Chemistry. The laboratory is accredited for the analysis of POPs according to ISO/IEC 17025 . Since 2011, it has participated twice a year in the intercomparison exercise "AMAP Ring Test for Persistent Organic Pollutants in Human Serum," which is organized by the Institut National de Santé Publique du Québec, Centre de Toxicologie du Québec (Canada), and has always met the performance acceptability criteria for PFOS and PFOA. 


\subsection{Statistical analysis}

Non-parametric Kruskal-Wallis and Mann-Whitney U tests and the Spearman correlation were used to investigate the statistical significance of the differences in the PFOS and PFOA serum concentrations between the groups and the possible correlations between PFOS and PFOA concentrations and variables from the questionnaires.

Information from questionnaires was incomplete for 70 women. As a consequence, the statistical analysis was performed on 479 of the 549 women enrolled.

\section{Results}

Anthropometric variables and socio-demographic characteristics of women enrolled, including the age distribution, body mass index (BMI), educational level, employment status, and residence, are shown in Table 1 . The mean age was 27.7 years. Most women (64\%) were less than 30 years old, which was a consequence of the difficulty in finding women who had never breastfed in the upper age class (30-41 years). Most subjects (71\%) had a normal BMI, with almost the same percentage (11 and 13, respectively) of women being under or over weight, and a small percent (4) were obese. Participants were well distributed according to their place of residence (56\% from urban and industrial areas and $44 \%$ from rural areas). Most women (93\%) had a medium to high educational level and were employed (66\%).

Table 2 summarizes the PFOS and PFOA concentrations stratified by age and by region. The data distributions were not normal and were skewed to the right for both compounds. The PFOS and PFOA median concentrations were 2.43 and $1.55 \mathrm{ng} \mathrm{g}^{-1}$, respectively. The P95 and maximum values were, respectively, 6.68 and $58.9 \mathrm{ng} \mathrm{g}^{-1}$ for PFOS and 3.46 and $9.99 \mathrm{ng} \mathrm{g}^{-1}$ for PFOA. The median serum concentrations of both compounds were lowest in Puglia and highest in Umbria.

The women who had the highest concentrations of PFOS resided in Piemonte (58.9 and $25.4 \mathrm{ng} \mathrm{g}^{-1}$ ) and in Umbria $\left(14.9 \mathrm{ng} \mathrm{g}^{-1}\right)$. The highest PFOA values were observed in two women residing in Umbria (9.99 and $7.99 \mathrm{ng} \mathrm{g}^{-1}$ ) and one in Piemonte (7.36 $\left.\mathrm{ng} \mathrm{g}^{-1}\right)$. Significant differences in concentration were observed between regions for PFOS and PFOA. In particular, women

Table 1

Anthropometric and socio-demographic characteristics of study participants.

\begin{tabular}{lll}
\hline & Mean (std dev) & $N(\%)$ \\
\hline Age (years) & $27.7(5.6)$ & \\
$20-29$ & & $172(36)$ \\
$30-41$ & $22.2(3.5)$ & \\
Body mass index $\left(\mathrm{kg} \mathrm{m}^{-2}\right)$ & $55(11)$ \\
Underweight $(\mathrm{BMI}<18.5)$ & $342(71)$ \\
Normal $(18.5<\mathrm{BMI}<25)$ & $64(13)$ \\
Overweight $(25<\mathrm{BMI}<30)$ & $18(4)$ \\
Obese (BMI $\geqslant 30)$ & \\
Residence & $267(56)$ \\
Urban or Industrial & $212(44)$ \\
Rural & \\
Educational level & \\
Primary school & $1(0)$ \\
Secondary school $(1 \mathrm{st}$ stage) & $34(7)$ \\
Secondary school $(2 \mathrm{nd}$ stage) & $248(52)$ \\
University & $195(41)$ \\
Employment status & \\
Employed & \\
Housewife & $311(66)$ \\
Student & $3(1)$ \\
Unemployed & $134(28)$ \\
\hline
\end{tabular}

from Umbria had significantly higher concentration of PFOS than did women from Trentino Alto Adige, Piemonte, Puglia, and Sicilia (Kruskall-Wallis Test, $p \ll 0.0001$ ). Women from Umbria had a significantly higher serum concentration of PFOA than did women from Piemonte, Lazio, Puglia, and Sicilia $(p \ll 0.0001)$.

Though the PFOS concentrations did not significantly differ among areas of different typology, the PFOA concentrations were significantly higher in women residing in urban and industrial areas than in women from rural areas $(p=0.017)$. The concentrations of the two compounds were strongly correlated $(p \ll 0.0001)$.

A statistically significant $(p<0.05)$ correlation was found between the concentrations of PFOS and PFOA and some of the variables from the questionnaires. In particular, a direct correlation with age was observed for PFOS ( $p \ll 0.0001$ ), but not for PFOA.

Additionally, women who had the highest educational level (university degree) showed significantly higher levels of PFOA than did women in the other education levels $(p<0.05)$. The same tendency was also observed for PFOS.

The contaminant concentrations did not differ significantly among the four categories of employment status, but students and employed subjects had higher serum levels of both compounds than did housewives and unemployed women; this difference was only marginally significant $(p<0.1)$ for PFOS. However, this result might have been affected by the low number of housewives and unemployed subjects $(N=29)$.

For each participant, the daily intake of specific food items was calculated based on the reported food consumption frequencies. The consumption figures derived from the questionnaires were in good agreement with figures reported by European Food Safety Authority (EFSA) for Italy for the same population group for the food categories considered (EFSA, 2008b). The results of the correlation analysis between the PFOS and PFOA serum concentrations and the consumption of selected food items are reported in Table 3, together with the results of the Mann Whitney $U$ test applied to PFOS and PFOA concentrations in consumers of locally produced food vs. consumers of food obtained from gross distribution or retail. The PFOS levels were significantly correlated with the consumption of beef ( $p \ll 0.01)$, pork ( $p \ll 0.01)$, fish $(p=0.05)$, and vegetables $(p \ll 0.01)$. As for PFOA, no statistically significant correlation was found with any of the food categories considered, except for a weak inverse correlation with chicken and ham consumption. The concentrations of both compounds were significantly higher $(p<0.05)$ in women who consumed beef, pork, lamb, ham and salami, fish and seafood from food retailers, whereas those of PFOA were higher $(p \leqslant 0.04)$ in women who consumed cheese, eggs, liver and vegetables from food retailers.

\section{Discussion}

This study represents the widest human biomonitoring study on PFOS and PFOA that has been carried out in Italy so far and, to the best of our knowledge, one of the widest carried out on women of reproductive age in the EU countries. To characterize exposure as a function of different geographical latitudes and environmental situations, the study design was based on a bifocal/trifocal approach. Consequently, in each of the six Regions considered, women residing in rural and/or mountainous areas and women residing in areas at a higher level of anthropic activity (urban and/or industrial areas) were included in the study.

The results show that a relevant exposure variability to both compounds exists among the Regions. Women from the Regions in southern Italy had the lowest $(p \ll 0.0001)$ levels of both PFOS and PFOA, followed by women from northern and central Italy. The Umbria Region seemed to be particularly affected by these contaminants. Although the reasons for the observed differences 
Table 2

Descriptive statistics of PFOS and PFOA concentrations ( $\left.\mathrm{ng} \mathrm{g}^{-1}\right)$ in serum samples stratified by age and Region of residence.

\begin{tabular}{|c|c|c|c|c|c|c|c|c|c|}
\hline & $\mathrm{N}$ & Minimum & P5 & P25 & Median & Mean & P75 & P95 & Maximum \\
\hline \multicolumn{10}{|l|}{ PFOS } \\
\hline All data & 479 & 0.34 & 0.95 & 1.73 & 2.43 & 3.06 & 3.45 & 6.68 & 58.9 \\
\hline Age $20-29$ & 307 & 0.34 & 0.904 & 1.70 & 2.31 & 2.92 & 3.11 & 6.04 & 58.9 \\
\hline Age $30-40$ & 172 & 0.61 & 1.02 & 1.77 & 2.72 & 3.31 & 4.02 & 6.73 & 25.4 \\
\hline Lazio & 61 & 0.97 & 1.49 & 1.94 & 2.56 & 3.23 & 3.49 & $7.78^{\mathrm{a}}$ & 14.1 \\
\hline Piemonte & 104 & 0.56 & 0.81 & 1.54 & 2.42 & 3.68 & 3.62 & $7.09^{a}$ & 58.9 \\
\hline Puglia & 85 & 0.34 & 0.74 & 1.34 & 1.71 & 1.96 & 2.56 & $3.45^{\mathrm{a}}$ & 5.50 \\
\hline Sicilia & 39 & 0.53 & 0.57 & 1.72 & 2.36 & 2.95 & 3.77 & $5.95^{\mathrm{a}}$ & 12.3 \\
\hline Trentino & 90 & 1.03 & 1.30 & 1.78 & 2.18 & 2.41 & 2.75 & $4.42^{\mathrm{a}}$ & 7.06 \\
\hline Umbria & 100 & 0.51 & 1.40 & 2.59 & 3.43 & 3.87 & 4.69 & $6.99^{a}$ & 14.9 \\
\hline \multicolumn{10}{|l|}{ PFOA } \\
\hline All data & 470 & $<0.1$ & 0.300 & 1.04 & 1.55 & 1.70 & 2.04 & 3.46 & 9.99 \\
\hline Age 20-29 & 300 & $<0.1$ & 0.310 & 1.06 & 1.54 & 1.66 & 2.01 & 3.20 & 9.99 \\
\hline Age $30-40$ & 170 & 0.12 & 0.28 & 0.97 & 1.59 & 1.76 & 2.14 & 4.00 & 7.36 \\
\hline Lazio & 61 & 0.20 & 0.23 & 0.41 & 1.52 & 1.47 & 2.17 & $3.23^{\mathrm{a}}$ & 3.91 \\
\hline Piemonte & 95 & $<0.1$ & 0.16 & 1.03 & 1.44 & 1.57 & 1.78 & $3.40^{\mathrm{a}}$ & 7.36 \\
\hline Puglia & 85 & 0.21 & 0.37 & 0.75 & 1.05 & 1.23 & 1.60 & $2.42^{\mathrm{a}}$ & 3.72 \\
\hline Sicilia & 39 & 0.45 & 0.46 & 0.88 & 1.32 & 1.49 & 1.84 & $3.17^{\mathrm{a}}$ & 5.22 \\
\hline Trentino & 90 & 0.59 & 1.08 & 1.37 & 1.71 & 1.73 & 1.94 & $2.82^{\mathrm{a}}$ & 4.05 \\
\hline Umbria & 100 & 0.33 & 0.54 & 1.49 & 2.04 & 2.41 & 2.81 & $5.57^{\mathrm{a}}$ & 9.99 \\
\hline
\end{tabular}

a P95 calculated on a number of subjects $N<160$ only provide a rough indication of high levels of serum concentrations EFSA (2008b).

Table 3

Correlation between PFOS and PFOA serum concentrations and consumption of selected food items and differences between concentrations in commercial vs. local food consumers. The values highlighted in bold are statistically significant $(p<0.05)$.

\begin{tabular}{|c|c|c|c|c|c|c|}
\hline & \multirow{3}{*}{$\begin{array}{l}\text { Number of consumers } \\
\mathrm{N}\end{array}$} & \multirow{3}{*}{$\begin{array}{l}\text { Percentage of consumers } \\
\text { of local food } \\
\text { (\%) }\end{array}$} & \multicolumn{2}{|c|}{$\begin{array}{l}\text { Spearman } \\
\text { correlation }\end{array}$} & \multicolumn{2}{|c|}{$\begin{array}{l}\text { Mann-Whitney } \\
\text { test }^{\mathrm{a}}\end{array}$} \\
\hline & & & PFOS & PFOA & PFOS & PFOA \\
\hline & & & $p$ & $p$ & $p$ & $p$ \\
\hline Milk and dairy products & 447 & 19 & 0.78 & 0.74 & 0.49 & $\mathbf{0 . 0 1}$ \\
\hline Milk & 372 & 3 & 0.37 & 0.33 & $-{ }^{\mathrm{b}}$ & - \\
\hline Yogurt & 317 & 1 & 0.19 & 0.16 & - & - \\
\hline Cheese & 355 & 22 & 0.13 & 0.25 & 0.27 & 0.01 \\
\hline Eggs & 396 & 37 & 0.59 & 0.20 & 0.30 & 0.01 \\
\hline Meat and meat products & 454 & 27 & $\ll \mathbf{0 . 0 1}{ }^{\mathrm{c}}$ & 0.84 & 0.99 & 0.12 \\
\hline Chicken & 423 & 24 & 0.71 & $0.04^{d}$ & 0.74 & 0.18 \\
\hline Beef & 365 & 19 & $\ll \mathbf{0 . 0 1}^{\mathrm{c}}$ & 0.24 & $<0.01$ & $\ll \mathbf{0 . 0 1}$ \\
\hline Liver & 68 & 19 & 0.71 & 0.18 & 0.21 & 0.04 \\
\hline Pork & 317 & 19 & $\ll \mathbf{0 . 0 1}^{\mathrm{C}}$ & 0.42 & 0.02 & 0.03 \\
\hline Lamb & 84 & 38 & 0.27 & 0.47 & $\ll \mathbf{0 . 0 1}$ & $\ll \mathbf{0 . 0 1}$ \\
\hline Ham, salami & 390 & 12 & 0.40 & $\mathbf{0 . 0 4} 4^{\mathrm{d}}$ & 0.04 & 0.03 \\
\hline Fish and seafood & 422 & 19 & 0.15 & 0.86 & $\ll \mathbf{0 . 0 1}$ & $\ll \mathbf{0 . 0 1}$ \\
\hline Fish & 415 & 19 & $0.05^{c}$ & 0.70 & $\ll \mathbf{0 . 0 1}$ & $\ll \mathbf{0 . 0 1}$ \\
\hline Seafood & 188 & 24 & 0.74 & 0.35 & $\ll \mathbf{0 . 0 1}$ & 0.02 \\
\hline Raw and/or cooked vegetables & 462 & 46 & $\ll \mathbf{0 . 0 1}{ }^{\mathrm{c}}$ & 0.34 & 0.13 & $\ll \mathbf{0 . 0 1}$ \\
\hline Cooked vegetables & 445 & 41 & $\ll \mathbf{0 . 0 1}{ }^{\mathrm{C}}$ & 0.48 & 0.08 & $\ll \mathbf{0 . 0 1}$ \\
\hline Raw vegetables & 449 & 47 & $\mathbf{0 . 0 1}{ }^{\mathrm{c}}$ & 0.25 & 0.14 & $\ll \mathbf{0 . 0 1}$ \\
\hline
\end{tabular}

${ }^{\text {a }}$ For all significant $(p<0.05)$ differences serum concentrations are higher in commercial food consumers.

b Mann-Whitney test was not applied when the percentage of consumers of local food was $<10 \%$.

c Direct correlation.

d Inverse correlation.

are difficult to identify, they are probably partly associated with differences in dietary habits and lifestyle.

Diet could play a particular role in PFOS exposure, as suggested by the significant correlation with meat and fish consumption. People from northern and central Italy traditionally have a diet that is richer in meat and meat products than do people from the south (Piccinelli et al., 2011), which was also confirmed by the questionnaires. Additionally, women residing in the northern Regions might be exposed to indoor dust containing higher levels of PFAS because of the greater use of treated carpets and textiles in colder regions (Kubwabo et al., 2005; Gewurtz et al., 2009).
Women residing in urban areas appear to be exposed to higher levels of PFOA. A comparatively higher exposure to PFOA was also observed for women with a high degree of education, who were more represented in urban areas in this study. Such an association between the higher levels of PFOA and a higher educational level has also been observed in other studies (Calafat et al., 2007; Melzer et al., 2010). These findings are not imputable to known variables, but lifestyle factors might play a role; particularly different dietary habits (i.e., a higher rate of consumption of food obtained from the retail level and of packaged fast-food) and more prolonged exposure to indoor environments, which are known to 
represent an important exposure pathway to PFAS, particularly to PFOA (Björklund et al., 2009; Haug et al., 2011).

Regarding the role of food consumption patterns, a significant correlation was detected between the PFOS concentrations and the intake of some meat products, fish and vegetables. These findings were, overall, consistent with the EFSA opinion (EFSA, 2012), which identified fish and meat as major contributors to dietary exposure to PFOS. Analysis of the association between concentrations of PFOS and PFOA and consumption of foodstuffs from the retail level or from local or their own production showed that retail food contributes to exposure more consistently than does locally produced food for most of the categories considered.

As already observed in a study conducted in 2007-2009 (Ingelido et al., 2010), the PFOS and PFOA serum concentrations in Italy are among the lowest recently observed in Europe (Kärrman et al., 2013). The reasons for this are unknown. Dietary intake is unanimously recognized as the major exposure pathway for PFAS. However, other factors, mainly dust ingestion, also contribute to overall exposure and account for a fraction that may be highly variable; they range from a few percentage units to approximately $40 \%$ in the worst case (Björklund et al., 2009). We hypothesize that the generally limited use of carpets in Italy might play a role, as suggested by some studies that showed a positive correlation between the percentage of home carpeting and PFAS internal dose (Kubwabo et al., 2005; Gewurtz et al., 2009), which was also confirmed by studies carried out in other Mediterranean countries (Ericson Jogsten et al., 2012), which, like Italy, have limited or no use of fitted carpets.

The serum concentrations assessed in the present study were compared with the concentrations observed in a study carried out in 2007-2009 (Ingelido et al., 2010) in 230 subjects (males and females, ages 20-65) residing in Rome and Brescia. The PFOS and PFOA levels appear to have significantly declined (MannWhitney $U$ test, $p \ll 0.001$ ), and the median values decreased from 6.31 to $2.43 \mathrm{ng} \mathrm{g}^{-1}$ for PFOS and from 3.59 to $1.55 \mathrm{ng} \mathrm{g}^{-1}$ for PFOA. For a better comparison that which was unaffected by differences in the subjects' age and sex, we selected two subgroups of subjects from the 2010 database. The first subgroup included men and women of the same age range as the present study (61 subject, 20-41 years), and the second included only a group of 24 women, whose characteristics were perfectly comparable to those of the women enrolled in the present study with respect to nulliparity and age. A comparison of the observed median concentrations in this study with those assessed for such subgroups showed a decline in the PFOS and PFOA concentrations of approximately $50 \%$ (Fig. 2).

A decline in PFOS serum concentrations over the last decade has been observed in other countries, including Germany (SchröterKermani et al., 2013), the U.S. (Olsen et al., 2012), Norway (Haug et al., 2009) and Sweden (Glynn et al., 2012). Further, a decrease in exposure to PFOA has been observed, although such a decrease is, on average, less marked with respect to PFOS (Glynn et al., 2012).
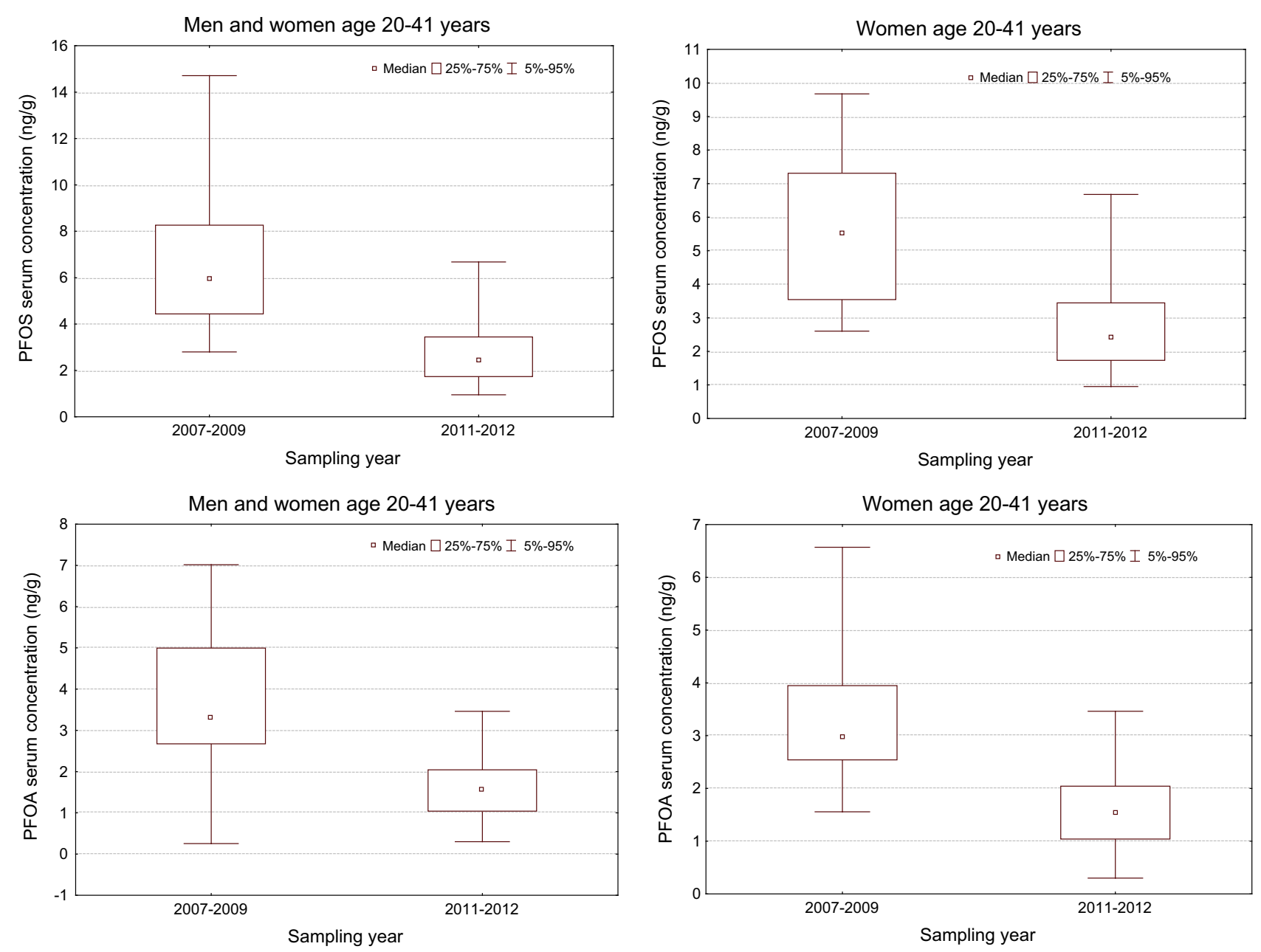

Fig. 2. Time trend of PFOS and PFOA serum concentrations $\left(\mathrm{ng} \mathrm{g}^{-1}\right)$ in men and women (left) and women only (right). 
Though the decline observed for PFOS is attributable to the production of this compound ceasing in Europe and North America, the less pronounced decline in exposure to PFOA could be due to its ongoing production and the use of PFOA and its precursors (Glynn et al., 2012).

It is worth noting that the decrease in serum concentrations observed for Italy is significantly sharper than generally observed for both compounds in other studies, most notably for PFOA.

The distributions of the observed PFOS and PFOA concentrations were markedly skewed to the right; the highest values were 10-25-fold higher than the median for PFOS, and 5-6-fold higher than the median for PFOA. Such exposure variability, together with evidence of placental transfer (approximately 30-80\% for PFOS, and $70-150 \%$ for PFOA, Fromme et al., 2010; Needham et al., 2011) as well as the observation that in a population residing in a polluted area (Mondal et al., 2012) both compounds were shown to accumulate in children via their mothers, indicates the need to continue monitoring exposure to PFAS in women of reproductive age.

This holds particularly true in the light of some recent toxicological findings (Grandjean and Budtz-Jørgensen, 2013) that showed that immunotoxicity in children might be associated with below average serum concentrations of PFOS and PFOA in women who were of reproductive age in recent population studies (Brede et al., 2010; Kärrman et al., 2013; Axmon et al., 2014; Berg et al., 2014) and below the median concentration values assessed in the present study.

\section{Acknowledgments}

We thank all of the women who participated in the Project as well as the doctors, nurses, midwives, and laboratory technicians who assisted for their generous collaboration. Special thanks for their essential technical support are due to Agnese Avaro (ASL TO5), Piera Berger (ASL TO5), Valentina Boselli (Ospedale S. Anna), Rosa Corvetti (ex ASL 4, Terni), Aldo Degaudenz (AVIS Trentino), De Luca Elena (ASL TO5), Mariangela Evangelio (ASL Taranto), Eliana Fuggetta (Università di Roma "Sapienza"), Simona Insogna (ASL Taranto), Katia Livolsi (Ospedale S. Anna), Alesia Longhi (ex ASL 4, Terni), Giuseppina Pezzarossi (APSS Trento), Presutto Patrizia (ASL TO5); Massimo Ripamonti (APSS Trento), Raffaella Romagna (Ospedale S. Anna), Grazia Maria Sponselli (ASL Taranto).

The study (Project n. LIFE08 ENV/IT/000423) was funded by the "Life Plus" financial instrument (EC, DG Environment), and cofunded by the Italian National Institute of Health, and by the Ministry of the Environment, Land and Territory.

\section{References}

Axmon, A., Axelsson, J., Jakobsson, K., Lindh, C.H., Jönsson, B.A.G., 2014. Time trends between 1987 and 2007 for perfluoroalkyl acids in plasma from Swedish women. Chemosphere 102, 61-67.

Barouki, R., Gluckman, P.D., Grandjean, P., Hanson, M., Heindel, J.J., 2012 Developmental origins of non-communicable disease: implications fo research and public health. Environ. Health 27, 11-42.

Berg, V., Nøst, T.H., Huber, S., Rylander, C., Hansen, S., Veyhe, A.S., Fuskevåg, O.M. Odland, J.Ø., Sandanger, T.M., 2014. Maternal serum concentrations of per- and polyfluoroalkyl substances and their predictors in years with reduced production and use. Environ. Int. 69, 58-66.

Björklund, J.A., Thuresson, K., De Wit, C.A., 2009. Perfluoroalkyl compounds (PFCs) in indoor dust: concentrations, human exposure estimates, and sources. Environ. Sci. Technol. 43, 2276-2281.

Brede, E., Wilhelm, M., Göenb, T., Müller, J., Rauchfuss, K., Kraft, M., Hölzer, J., 2010 Two-year follow-up biomonitoring pilot study of residents and controls PFC plasma levels after PFOA reduction in public water system in Arnsberg, Germany. Int. J. Hyg. Environ. Health 213, 217-223.

Calafat, A.M., Kuklenyik, Z., Reidy, J.A., Caudill, S.P., Tully, J.S., Needham, L.L., 2007. Serum concentrations of 11 polyfluoroalkyl compounds in the U.S. population: data from the national health and nutrition examination survey (NHANES) Environ. Sci. Technol. 41, 2237-2242.
DeWitt, J.C., Peden-Adams, M.M., Keller, J.M., Germolec, D.R., 2012. Immunotoxicity of perfluorinated compounds: recent developments. Toxicol. Pathol. 40, 300311

Directive 2006/122/EC of the European Parliament and of the Council of 12 December 2006 amending for the 30th time Council Directive 76/769/EEC on the approximation of the laws, regulations and administrative provisions of the Member States relating to restrictions on the marketing and use of certain dangerous substances and preparations (perfluorooctane sulfonates).

EFSA, 2008a. Perfluorooctane sulfonate (PFOS), perfluorooctanoic acid (PFOA) and their salts. Scientific opinion of the panel on contaminants in the food chain. EFSA J. 653, 1-131 (Question N_EFSA-Q-2004-163).

EFSA, 2008b. Guidance document for the use of the concise European food consumption database in exposure assessment. EFSA/DATEX/2008/01. Parma, Italy (17.03.08)

EFSA, 2012. Perfluoroalkylated substances in food: occurrence and dietary exposure. EFSA J. 10, 2743.

Ericson Jogsten, I., Nadal, M., van Bavel, B., Lindström, G., Domingo, J.L., 2012. Per- and polyfluorinated compounds (PFCs) in house dust and indoor air in Catalonia, Spain: implications for human exposure. Environ. Int. 39, 172180.

Fei, C., McLaughlin, J.K., Lipworth, L., Olsen, J., 2009. Maternal levels of perfluorinated chemicals and subfecundity. Hum. Reprod. 24, 1200-1205.

Fromme, H., Mosch, C., Morovitz, M., Alba-Alejandre, I., Boehmer, S., Kiranoglu, M., Faber, F., Hannibal, I., Genzel-Boroviczény, O., Koletzko, B., Völkel, W., 2010. Preand postnatal exposure to perfluorinated compounds (PFCs). Environ. Sci. Technol. 44, 7123-7129.

Gewurtz, S.B., Bhavsar, S.P., Crozier, P.W., Diamond, M.L., Helm, P.A., Marvin, C.H., Reiner, E.J., 2009. Perfluoroalkyl contaminants in window film: indoor/outdoor, urban/rural, and winter/summer contamination and assessment of carpet as a possible source. Environ. Sci. Technol. 43, 7317-7323.

Glynn, A., Berger, U., Bignert, A., Ullah, S., Aune, M., Lignell, S., Darnerud, P.O., 2012. Perfluorinated alkyl acids in blood serum from primiparous women in Sweden: serial sampling during pregnancy and nursing, and temporal trends 1996-2010. Environ. Sci. Technol. 46, 9071-9079.

Grandjean, P., Budtz-Jørgensen, E., 2013. Immunotoxicity of perfluorinated alkylates: calculation of benchmark doses based on serum concentrations in children. Environ. Health 12, 35.

Haug, L.S., Thomsen, C., Becher, G., 2009. Time trends and the influence of age and gender on serum concentrations of perfluorinated compounds in archived human samples. Environ. Sci. Technol. 43, 2131-2136.

Haug, L.S., Huber, S., Becher, G., Thomsen, C., 2011. Characterisation of human exposure pathways to perfluorinated compounds - comparing exposure estimates with biomarkers of exposure. Environ. Int. 37, 687-693.

IARC, 2008. World Health Organization, International Agency for Research on Cancer. IARC, Monographs on the evaluation of carcinogenic risks to humans. Report of the Advisory Group to Recommend Priorities for IARC Monographs during 2010-2014, 17-20 June 2008. Lyon France.

Ingelido, A.M., Marra, V., Abballe, A., Valentini, S., Iacovella, N., Barbieri, P., Porpora, M.G., di Domenico, A., De Felip, E., 2010. Perfluorooctanesulfonate and perfluorooctanoic acid exposures of the Italian general population. Chemosphere 80, 1125-1130.

Inoue, K., Okada, F., Ito, R., Kato, S., Sasaki, S., Nakajima, S., Uno, A., Saijo, Y., Sata, F., Yoshimur, Y., Kishi, R., Nakazawa, H., 2004. Perfluorooctane sulfonate (PFOS) and related perfluorinated compounds in human maternal and cord blood samples: assessment of PFOS exposure in a susceptible population during pregnancy. Environ. Health Persp. 112, 1204-1207.

Kärrman, A., Davies, J., Salihovic, S., 2013. PFAAs in matched milk and serum from primipara women. Report of the Swedish Environmental Protection Agency (Naturvårdsverket) NV-12526-11.

Kristensen, S.L., Ramlau-Hansen, C.H., Ernst, E., Olsen, S.F., Bonde, J.P., Vested, A., Halldorsson, T.I., Becher, G., Haug, L.S., Toft, G., 2013. Long-term effects of prenatal exposure to perfluoroalkyl substances on female reproduction. Hum. Reprod. 28, 3337-3348.

Kubwabo, C., Stewart, B., Zhu, J., Marro, L., 2005. Occurrence of perfluorosulfonates and other perfluorochemicals in dust from selected homes in the city of Ottawa, Canada. J. Environ. Monit. 7, 1074-1078.

Lau, C., 2012. Perfluoroalkyl acids: recent research highlights. Reprod. Toxicol. 33, 405-409.

Mannetje, A., Coakley, J., Mueller, J.F., Harden, F., Toms, L.M., Douwes, J., 2012. Partitioning of persistent organic pollutants (POPs) between human serum and breast milk: a literature review. Chemosphere 89, 911-918.

Melzer, D., Rice, N., Depledge, M.H., Henley, W.E., Galloway, T.S., 2010. Association between serum perfluorooctanoic acid (PFOA) and thyroid disease in the U.S. national health and nutrition examination survey. Environ. Health Persp. 118, 686-692.

Mondal, D., Lopez-Espinosa, M.J., Armstrong, B., Stein, C.R., Fletcher, T., 2012. Relationships of perfluorooctanoate and perfluorooctane sulfonate serum concentrations between mother and child pairs in a population with perfluorooctanoate exposure from drinking water. Environ. Health Persp. 120, $752-757$.

Needham, L.L., Grandjean, P., Heinzow, B., Jorgensen, P.J., Nielsen, F., Patterson Jr., D.G., Sjodin, A., Turner, W.E., Weihe, P., 2011. Partition of environmental chemicals between maternal and fetal blood and tissues. Environ. Sci. Technol. 45, 1121-1126.

Olsen, G.W., Lange, C.C., Ellefson, M.E., Mair, D.C., Church, T.R., Goldberg, C.L., Herron, R.M., Medhdizadehkashi, Z., Nobiletti, J.B., Rios, J.A., Reagen, W.K., Zobel, 
L.R., 2012. Temporal trends of perfluoroalkyl concentrations in American Red Cross adult blood donors, 2000-2010. Environ. Sci. Technol. 46, 6330-6338.

Piccinelli, R., Arcella, D., Buonocore, P., Capriotti, M., D’Addezio, L., Le Donne, C., Mistura, L., Pettinelli, A., Sette, S., Turrini, A., Leclercq, C., 2011. L'indagine nazionale sui consumi alimentari in Italia INRAN-SCAI 2005-06. Parte B3 - I consumi in termini di gruppi e sottogruppi alimentari per aree geografiche $(\mathrm{g} /$ die) - APPENDICE 6.B3_Carne, insaccati e sostituti della carne. Osservatorio Consumi Alimentari, INRAN.

Post, G.B., Cohn, P.D., Cooper, K.R., 2012. Perfluorooctanoic acid (PFOA), an emerging drinking water contaminant: a critical review of recent literature. Environ. Res. 116, 93-117.

Schröter-Kermani, C., Müller, J., Jürling, H., Conrad, A., Schulte, C., 2013. Retrospective monitoring of perfluorocarboxylates and perfluorosulfonates in human plasma archived by the German Environmental Specimen Bank. Int. J. Hyg. Environ. Health 216, 633-640.

Vestergaard, S., Nielsen, F., Andersson, A.M., Hjøllund, N.H., Grandjean, P., Andersen, H.R., Jensen, T.K., 2012. Association between perfluorinated compounds and time to pregnancy in a prospective cohort of Danish couples attempting to conceive. Hum. Reprod. 27, 873-880.

White, S.S., Fenton, S.E., Hines, E.P., 2011. Endocrine disrupting properties of perfluorooctanoic acid. J. Steroid Biochem. Mol. Biol. 127, 16-26.

Whitworth, K.W., Haug, L.S., Baird, D.D., Becher, G., Hoppin, J.A., Skjaerven, R., Thomsen, C., Eggesbo, M., Travlos, G., Wilson, R., Cupul-Uicab, L.A., Brantsaeter A.L., Longnecker, M.P., 2012. Perfluorinated compounds in relation to birth weight in the Norwegian mother and child cohort study. Am. J. Epidemiol. 175 1209-1216.

Wilhelm, M., Bergmann, S., Dieter, H.H., 2010. Occurrence of perfluorinated compounds (PFCs) in drinking water of North Rhine-Westphalia, Germany and new approach to assess drinking water contamination by shorter-chained C4-C7 PFCs. Int. J. Hyg. Environ. Health 213, 224-232.

Zhai, A., Axt, J., Hamilton, E.C., Koehler, E., Lovvorn 3rd., H.N., 2012. Assessing gonadal function after childhood ovarian surgery. J. Pediatr. Surg. 47, 1272 1279. 